\title{
Clinical manifestations of pneumonia according to the causative organism in patients in the intensive care unit
}

Jung-Kyu Lee, Jinwoo Lee, Young Sik Park, Chang Hoon Lee, Jae-Joon Yim, Chul-Gyu Yoo, Young Whan Kim, Sung Koo Han, and Sang-Min Lee

Division of Pulmonary and Critical Care Medicine, Department of Internal Medicine, Seoul National University Hospital, Seoul, Korea

Received: May 14, 2014

Revised : June 23, 2014

Accepted: October 8, 2014

Correspondence to

Sang-Min Lee, M.D.

Division of Pulmonary and

Critical Care Medicine,

Department of Internal Medicine,

Seoul National University

Hospital, 101 Daehak-ro,

Jongno-gu, Seoul 03080, Korea

Tel: +82-2-2072-0833

Fax: +82-2-762-9662

E-mail: sangmin2@snu.ac.kr
Background/Aims: Whether the causative organism influences the clinical course of pneumonia in the intensive care unit (ICU) is controversial. We assessed the clinical manifestations and prognosis of pneumonia according to the causative pathogens in patients in a medical ICU.

Methods: A retrospective observational study was performed in a medical ICU. Among 242 patients who were admitted to the ICU, 103 who were treated for pneumonia were analyzed.

Results: The causative pathogen was identified in 50 patients (49.0\%); 22 patients (21.6\%) had multidrug-resistant (MDR) pathogens. The distribution of causative micro-organisms was Staphylococcus aureus (20\%), Pseudomonas species (16\%), Klebsiella pneumoniae (14\%), and Acinetobacter baumannii (12\%). No significant difference in ICU mortality rate, duration of ICU stay, duration of mechanical ventilation, or frequencies of re-intubation and tracheostomy were detected based on the identification of any pathogen. In sub-analyses according to the pneumonia classification, the number of pathogens identified did not differ between pneumonia types, and a higher incidence of identified MDR pathogens was detected in the hospital-acquired pneumonia group than in the community-acquired or healthcare-acquired pneumonia groups. However, the clinical outcomes of pneumonia according to identification status and type of pathogen did not differ significantly between the groups.

Conclusions: Neither the causative micro-organism nor the existence of MDR pathogens in critically ill patients with pneumonia was associated with the clinical outcome of pneumonia, including ICU mortality. This result was consistent regardless of the pneumonia classification.

Keywords: Pneumonia; Pathogenicity; Drug resistance, multiple; Intensive care units

\section{INTRODUCTION}

Pneumonia is the second most common cause of in-hospital infection. Pneumonia is very common in the intensive care unit (ICU) setting and can be fatal. The inci- dence of pneumonia is about $17 \%$ in the medical ICU [1] but can be 6 to 20 times higher in mechanically ventilated patients [2]. The mortality rate of hospital-acquired pneumonia (HAP) depends on the clinical situation, but can range from $20 \%$ to $50 \%[3,4]$. Determining the prog- 
nosis of pneumonia is important for anticipating the disease course and establishing a proper management plan.

The causative micro-organism can influence the clinical presentation, outcome of antibiotic therapy, and prognosis in patients with pneumonia. However, whether the causative organism influences the clinical course of pneumonia in the ICU is controversial. Identifying a pathogen by culture can be a poor prognostic factor [5], and infection with multidrug-resistant (MDR) pathogens such as Acinetobacter baumannii or methicillin-resistant Staphylococcus aureus (MRSA) is a risk factor for hospital mortality [6-8]. However, one study reported that the pathogen classification or the existence of MDR pathogens does not affect the mortality rate after adjusting for the effect of antibiotics [9]. Although one study has reported the epidemiology and causative micro-organisms of pneumonia in Korea [10], the prognosis of pneumonia according to the causative micro-organism in the ICU is not well known. Thus, in this study, we elucidated the clinical manifestations and prognosis of patients with pneumonia according to the causative pathogen in the medical ICU.

\section{METHODS}

\section{Study design and participants}

A retrospective observational study was performed in the medical ICU of the Seoul National University Hospital between January 2011 and August 2011. We included patients with pneumonia treated in the medical ICU. Patients were enrolled if they had pneumonia on admission or developed pneumonia during their ICU stay. A total of 242 patients were admitted and treated in the medical ICU over the study period; in addition, data from 102 patients with pneumonia were analyzed retrospectively.

Pneumonia was clinically classified based on the American Thoracic Society/Infectious Disease Society of America guidelines [11,12]. According to these guidelines, healthcare-associated pneumonia (HCAP) was defined as pneumonia in any patient admitted to an acute care hospital for $\geq 2$ days within 90 days of the infection; who resided in a nursing home or long-term care facility; who received recent intravenous antibiotic therapy, chemotherapy, or wound care within 30 days of onset of the current infection; or who attended a hospital or hemodialysis clinic within 30 days. HAP was defined as pneumonia that developed $\geq 48$ hours after admission. Community-acquired pneumonia (CAP) was defined as pneumonia that did not meet any of the HCAP and HAP criteria.

The causative organisms were considered to be micro-organisms that had been isolated from specimens, including blood, bronchoalveolar lavage fluid, bronchial wash, and pleural effusion, and which grew to greater than a threshold concentration in quantitative cultures. Specimens obtained by endotracheal aspiration or in sputum expectorant were evaluated as appropriate using counts of white blood cells and epithelial cells, and the micro-organisms identified were considered the causative pathogen. Growth below the threshold was considered to be caused by colonization or contamination. Legionella and Streptococcus urinary antigen tests, viral polymerase chain reaction, and antigen tests were also used to identify the pathogens. Pathogens may have been confirmed in additional samples after a patient developed pneumonia, but a secondary infection due to a hospital- or ICU-acquired pathogen was ruled out. Specimens sampled within 3 days after a patient developed pneumonia were considered significant.

Empirical antibiotic regimens to treat pneumonia were reviewed, and their response and relevance to subsequent changes in the antibiotic regimen were analyzed. The initial empirical antibiotic regimen was administered according to the American Thoracic Society/ Infectious Disease Society of America guidelines [11,12]. Initial non-responders to empirical antibiotics were defined as cases in which the initial antibiotic was changed due to expansion or a switch in the antibiotic spectrum due to persistence or worsening of the clinical course within 1 week after developing pneumonia. This did not include cases in which empirical antibiotics were changed to the targeted regimen based on drug susceptibility in clinically improving patients.

We analyzed ICU mortality, duration of ICU stay, duration of mechanical ventilation, frequency of re-intubation, and frequency of tracheostomy as clinical outcomes of pneumonia. These outcomes were compared between patients with CAP, HCAP, and HAP.

This retrospective data collection was approved by the 
Institutional Review Board of Seoul National University (IRB no: H-1208-150-424) and was performed in accordance with the Declaration of Helsinki.

\section{Statistical analysis}

Data are presented as means and standard deviations for continuous variables and as numbers (percentages) for categorical variables. We analyzed baseline characteristics, clinical outcomes, and prognoses according to specific pathogens using the Kruskal-Wallis test. We used the Mann-Whitney test to compare data for prognosis according to whether a pathogen or MDR pathogen was identified. Associations between prognostic factors and survival rate were investigated using the Cox proportional-hazard analysis after adjusting for age, sex, Acute Physiology and Chronic Health Evaluation (APACHE) II score [13], Sequential Organ Failure Assessment (SOFA) score [14], Charlson comorbidity index [15], pneumonia severity index [16], the identification of the pathogen or MDR pathogen, and the pneumonia classification. Likelihood ratio tests were used to examine the goodness of fit of the model, and no lack of fit was found. Adjusted odd ratios (aOR) are presented with 95\% confidence intervals (CIs). A $p<0.05$ was considered significant. Data were analyzed using SPSS version 18 (SPSS Inc., Chicago, IL, USA).

\section{RESULTS}

\section{Baseline characteristics and clinical outcomes of the patients}

During the study period, 242 patients were admitted to the ICU; 102 had pneumonia, and all were eligible for this study. The study population was comprised of 20 patients (19.6\%) with CAP, 26 (25.5\%) with HCAP, and 56

Table 1. Baseline characteristics of patients with pneumonia treated in the intensive care unit

\begin{tabular}{|c|c|c|c|c|}
\hline Characteristic & $\begin{array}{l}\text { Overall } \\
(\mathrm{n}=102)\end{array}$ & $\begin{array}{l}\text { Pathogen not identified } \\
\qquad(\mathrm{n}=52)\end{array}$ & $\begin{array}{l}\text { Pathogen identified } \\
\qquad(\mathrm{n}=50)\end{array}$ & $p$ value \\
\hline Age, yr & $64.1 \pm 14.3$ & $66.0 \pm 11.5$ & $62.1 \pm 16.5$ & 0.410 \\
\hline Male sex & $77(75 \cdot 5)$ & $43(82.7)$ & $34(68)$ & 0.086 \\
\hline APACHE II score & $26.1 \pm 9.4$ & $26.0 \pm 9.4$ & $26.2 \pm 9.5$ & 0.815 \\
\hline SOFA score & $8.9 \pm 4.4$ & $8.5 \pm 3.7$ & $9.4 \pm 5.1$ & 0.541 \\
\hline Pneumonia severity index & $142.7 \pm 39.7$ & $142.3 \pm 41.6$ & $143.1 \pm 38.1$ & 0.723 \\
\hline Charlson comorbidity index & $3.7 \pm 2.5$ & $3.7 \pm 2.7$ & $3.6 \pm 2.4$ & 0.837 \\
\hline \multicolumn{5}{|l|}{ Comorbidity } \\
\hline Chronic lung disease & $29(28.4)$ & $17(32.7)$ & $12(24)$ & 0.333 \\
\hline Chronic heart disease & $8(7.8)$ & $3(5.8)$ & $5(10)$ & 0.429 \\
\hline Chronic liver disease & $17(16.7)$ & $6(11.5)$ & $11(22)$ & 0.158 \\
\hline Chronic renal disease & $15(14 \cdot 7)$ & $5(9.6)$ & $10(20)$ & 0.141 \\
\hline Solid tumor & $42(41.2)$ & $28(53.8)$ & $14(28)$ & 0.008 \\
\hline Diabetes mellitus & $32(31.4)$ & $12(23.1)$ & $20(40)$ & 0.067 \\
\hline Hematologic malignancy & $18(17.6)$ & $6(11.5)$ & $12(24)$ & 0.101 \\
\hline \multicolumn{5}{|l|}{ Pneumonia type } \\
\hline Community-acquired pneumonia & $20(19.6)$ & $11(21.2)$ & $9(18)$ & 0.690 \\
\hline Healthcare-associated pneumonia & $26(25 \cdot 5)$ & $15(28.8)$ & $11(22)$ & 0.430 \\
\hline Hospital-acquired pneumonia & $56(54 \cdot 9)$ & $26(50)$ & $30(60)$ & 0.313 \\
\hline MDR pathogen & $22(21.6)$ & 0 & $22(44)$ & $<0.001$ \\
\hline
\end{tabular}

Values are presented as mean \pm SD or number (\%).

APACHE, Acute Physiology and Chronic Health Evaluation; SOFA, Sequential Organ Failure Assessment; MDR, multidrug-resistant. 
(54.9\%) with HAP.

The baseline characteristics and clinical outcomes of the patients treated for pneumonia in the ICU are presented in Table 1. Mean age of the entire population was $64.1 \pm 14.3$ years, and $75.5 \%$ were men. No significant differences were found between the CAP, HCAP, and HAP groups for age, sex distribution, or severity as evaluated by the APACHE II score and the SOFA score. However, the Charlson comorbidity index and pneumonia severity index scores were significantly higher in the HCAP and HAP groups than in the CAP group; the scores did not differ between the HCAP and HAP groups.

The causative pneumonia pathogens were identified in 50 patients (49.0\%). No significant differences in baseline characteristics were observed according to the pathogens identified except for a history of solid tumor within 5 years and the presence of a MDR pathogen.

\section{Distribution of causative pathogens and clinical outcomes}

Among 50 cases in which a pathogen was identified, sputum (24, 48\%), blood (17,34\%), endotracheal aspirate (12, 24\%), bronchoalveolar lavage (4,8\%), serology (4, $8 \%)$, and bronchial wash $(2,4 \%)$ were the specimens in which pathogens were confirmed. The pathogens causing pneumonia were identified in nine patients (45\%) with CAP, in 11 (42.3\%) with HCAP, and in 30 (53.6\%) with HAP. No significant difference in the incidence of pathogens was observed among the pneumonia types after adjusting for culture frequency. The incidence of pneumonia with a MDR pathogen was 22 patients (21.6\%) in the entire population. The incidence was sig-

Table 2. Microetiological diagnoses and prognoses of pneumonia when the causative pathogen was identified $(n=50)$

\begin{tabular}{|c|c|c|c|c|c|}
\hline Variable & Frequency & MDR proportion & ICU mortality, \% & ICU LOS, day & MV duration, day \\
\hline \multicolumn{6}{|l|}{ Gram-positive bacteria } \\
\hline Staphylococcus aureus & $10(20)$ & $6(60)$ & $4(40)$ & $13.0 \pm 8.8$ & $7 \cdot 7 \pm 7 \cdot 4$ \\
\hline Streptococcus pneumoniae & $3(6)$ & o & $1(33 \cdot 3)$ & $40 \pm 17.6$ & $22.7 \pm 26.7$ \\
\hline Enterococcus faecium & $1(2)$ & $1(100)$ & 1 & 5 & 5 \\
\hline \multicolumn{6}{|l|}{ Gram-negative bacteria } \\
\hline \multicolumn{6}{|l|}{ Enterobacteriaceae } \\
\hline Klebsiella pneumoniae & $7(14)$ & $2(28.6)$ & $4(57 \cdot 1)$ & $7 \cdot 4 \pm 5 \cdot 5$ & $2.6 \pm 3.0$ \\
\hline Acinetobacter baumannii & $6(12)$ & $5(83 \cdot 3)$ & $3(50)$ & $12.7 \pm 15.5$ & $12.8 \pm 15.5$ \\
\hline Escherichia coli & $3(6)$ & $2(66.7)$ & $1(33 \cdot 3)$ & $11.0 \pm 10.1$ & $3 \cdot 3 \pm 4 \cdot 9$ \\
\hline Enterobacter aerogenes & $2(4)$ & $1(50)$ & 0 & $13.0 \pm 17.0$ & $12.5 \pm 17.7$ \\
\hline \multicolumn{6}{|c|}{ Nonfermentative gram-negative bacilli } \\
\hline Pseudomonas species & $8(16)$ & $5(62.5)$ & $3(37.5)$ & $13.1 \pm 8.0$ & $5 \cdot 9 \pm 8.0$ \\
\hline Stenotrophomonas maltophilia & $2(4)$ & $2(100)$ & $2(100)$ & $4.0 \pm 1.4$ & $3.5 \pm 2.1$ \\
\hline \multicolumn{6}{|l|}{ Mycobacterium } \\
\hline Mycobacterium tuberculosis & $1(2)$ & 0 & o & 52 & 9 \\
\hline Non-tuberculosis Mycobacterium & $1(2)$ & 0 & $1(100)$ & 1 & 0 \\
\hline \multicolumn{6}{|l|}{ Fungi } \\
\hline Aspergillus species & $5(10)$ & 0 & $3(60)$ & $23.2 \pm 29.8$ & $19.0 \pm 29.1$ \\
\hline \multicolumn{6}{|l|}{ Virus } \\
\hline Cytomegalovirus & $4(8)$ & 0 & $2(50)$ & $20.5 \pm 14.6$ & $12.5 \pm 8.2$ \\
\hline \multicolumn{6}{|l|}{ Atypical pathogen } \\
\hline Pneumocystis jiroveci & $2(2.0)$ & 0 & $1(50)$ & $14.5 \pm 6.4$ & $13.5 \pm 7.8$ \\
\hline Mycoplasma species & $1(1.0)$ & o & $1(100)$ & 15 & 15 \\
\hline
\end{tabular}

Values are presented as number $(\%)$ or mean $\pm \mathrm{SD}$.

MDR, multidrug-resistant; ICU, intensive care unit; LOS, length of stay; MV, mechanical ventilation. 
nificantly higher in the HAP group than in the HCAP and CAP groups (HAP, 32.1\%; HCAP, 11.5\%; CAP, 5.0\%; $p=0.015)$, but was not different between the CAP and HCAP groups. The MDR micro-organisms were: carbapenem-resistant A. baumannii, MRSA, coagulase-negative Staphylococcus, vancomycin-resistant enterococci, extended-spectrum b-lactamase-producing Escherichia coli, Klebsiella, and MDR Pseudomonas species.

Table 2 shows the distribution of specific pathogens and the respective prognoses. $S$. aureus was the most frequently isolated pathogen among all patients in all groups (10, 20\%). Pseudomonas species were detected in eight patients (16\%), Klebsiella pneumoniae in seven (14\%), and A. baumannii in six (12\%). No significant difference was observed in the clinical outcomes between specific pathogens.

\section{Regimen and response to empirical antibiotic treat- ment}

The empirical antibiotics and their responses are shown in Table 3. The most commonly used antibiotics as an empirical regimen, including combination therapy, were $\beta$-lactam $/ \beta$-lactamase inhibitors $(62,60.8 \%)$, fol-

Table 3. Empirical antibiotic regimen and response $(n=102)$

\begin{tabular}{lc}
\hline Variable & No. $(\%)$ \\
\hline Empirical antibiotic treatment & \\
$\beta$-Lactam/ $\beta$-lactamase inhibitor & $62(60.8)$ \\
\hline Quinolone & $57(55.9)$ \\
\hline Carbapenem & $20(19.6)$ \\
\hline Vancomycin & $19(18.6)$ \\
\hline Cephalosporin & $15(14.7)$ \\
\hline Trimethoprim/sulfamethoxazole & $14(13.7)$ \\
\hline Antifungal agents & $10(9.8)$ \\
\hline Antiviral agents & $5(4.9)$ \\
\hline Macrolides & $5(4.9)$ \\
\hline Colistin & $3(2.9)$ \\
\hline $\begin{array}{l}\text { Initial responder to empirical antibiotic } \\
\text { therapy }\end{array}$ & $59(57.8)$ \\
\hline $\begin{array}{l}\text { Antibiotics response in cases with identified } \\
\text { pathogens (n = 50) }\end{array}$ & \\
\hline Initially appropriate antibiotics & $30(60)$ \\
\hline Subsequent change to targeted antibiotics & $28(56)$ \\
\hline $\begin{array}{l}\text { Appropriate change according to drug } \\
\text { susceptibility }\end{array}$ & $25(89.3)$ \\
\hline
\end{tabular}

lowed by quinolones $(57,55.9 \%)$. About $58 \%$ of the patients responded initially to the empirical antibiotic regimen. Appropriate antibiotics were initially used in $60 \%$ of cases when a retrospective review was performed considering pathogen drug susceptibility and the antibiotic spectrum in cases in which the pathogen was identified. In these cases, a subsequent change to the targeted antibiotic occurred in $56 \%$ of cases, and most (89.3\%) were appropriate considering the drug susceptibility of the pathogen.

\section{Clinical outcomes according to identification of the causative organism and pneumonia classification}

The prognoses of pneumonia based on identifying the pathogens are shown in Table 4. The ICU mortality rate was $47.1 \%$ in the entire study population, and did not differ according to whether the pathogen was identified (unidentified pathogen $46.2 \%$ vs. identified pathogen $48.0 \%, p=0.853)$. Other clinical outcomes such as duration of ICU stay, need for and duration of mechanical ventilation, and frequencies of re-intubation and tracheostomy were different based on whether the pathogen was identified. These results were consistent with cases in which MDR pathogens were identified.

A subgroup analysis was performed according to pneumonia type, and the clinical outcomes did not differ significantly between the groups.

\section{Prognostic factors for mortality}

A Cox proportional-hazard analysis was performed to evaluate the relative risk of ICU mortality caused by pneumonia, and the data are presented in Table 5. The ICU mortality rate was significantly higher in the group with higher APACHE II and SOFA scores in both univariate and multivariate analyses ([APACHE II score: aOR, 1.07; 95\% CI, 1.03 to 1.11]; [SOFA score: aOR, 1.26; 95\% CI, 1.14 to 1.38]). Age was also a significant prognostic factor for ICU mortality in the multivariate analysis (aOR, 1.04; 95\% CI, 1.01 to 1.07). The pneumonia severity index was significantly associated with ICU mortality in the univariate analysis, but significance was lost after adjustment. Age, sex, the Charlson comorbidity index, identification of the pathogen or MDR pathogen, initial responder to empirical antibiotic therapy, and pneumonia classification were not significant prognostic indicators in the ICU mortality evaluation. 
Table 4. Clinical outcomes and prognoses of pneumonia according to whether the pathogen was identified

\begin{tabular}{|c|c|c|c|c|c|c|}
\hline \multirow{2}{*}{ Variable } & \multicolumn{3}{|c|}{ Pathogens } & \multicolumn{3}{|c|}{ MDR pathogens } \\
\hline & Absence & Presence & $p$ value & Absence & Presence & $p$ value \\
\hline Overall $(n=102)$ & 52 & 50 & & 80 & 22 & \\
\hline ICU mortality & $24(46.2)$ & $24(48)$ & 0.853 & $38(47 \cdot 5)$ & $10(45 \cdot 5)$ & 0.865 \\
\hline ICU length of stay, day & $19.0 \pm 26.8$ & $15 \cdot 4 \pm 15 \cdot 4$ & 0.176 & $18.7 \pm 24.3$ & $11.8 \pm 7.6$ & 0.305 \\
\hline MV duration, day & $15 \cdot 5 \pm 27 \cdot 3$ & $10.1 \pm 13.7$ & 0.060 & $14 \cdot 4 \pm 24 \cdot 1$ & $7 \cdot 0 \pm 7.1$ & 0.062 \\
\hline Re-intubation & $9(17 \cdot 3)$ & $5(10)$ & 0.286 & $13(16.3)$ & $1(4 \cdot 5)$ & 0.189 \\
\hline Tracheostomy & $13(25)$ & $11(22)$ & 0.722 & $18(22.5)$ & $6(27 \cdot 3)$ & 0.777 \\
\hline CAP group $(n=20)$ & 11 & 9 & & 19 & 1 & \\
\hline ICU mortality & $2(18.2)$ & $5(55 \cdot 6)$ & 0.160 & $6(31.6)$ & $1(100)$ & 0.350 \\
\hline ICU length of stay, day & $14.9 \pm 10.1$ & $15.9 \pm 16.0$ & 0.909 & $15 \cdot 4 \pm 13.1$ & 15 & 0.794 \\
\hline MV duration, day & $11.5 \pm 10.4$ & $7 \cdot 3 \pm 7 \cdot 7$ & 0.340 & $9.3 \pm 9.4$ & 15 & 0.257 \\
\hline Re-intubation & $3(27 \cdot 3)$ & $2(22.2)$ & 1.000 & $5(26.3)$ & o & 1.000 \\
\hline Tracheostomy & $4(36.4)$ & 0 & 0.094 & $4(21.1)$ & o & 1.000 \\
\hline HCAP group $(n=26)$ & 15 & 11 & & 23 & 3 & \\
\hline ICU mortality & $7(46.7)$ & $2(18.2)$ & 0.217 & $9(39.1)$ & $\mathrm{o}$ & 0.294 \\
\hline ICU length of stay, day & $15 \cdot 5 \pm 8.3$ & $18.0 \pm 18.6$ & 0.612 & $17.4 \pm 13.9$ & $9.7 \pm 6.7$ & 0.377 \\
\hline MV duration, day & $11.9 \pm 7.8$ & $11.4 \pm 14.9$ & 0.275 & $12.6 \pm 11.4$ & $5 \cdot 0 \pm 5.6$ & 0.137 \\
\hline Re-intubation & $3(20)$ & $1(9.1)$ & 0.614 & $4(17 \cdot 4)$ & $\mathrm{o}$ & 1.000 \\
\hline Tracheostomy & $1(6.7)$ & $2(18.2)$ & 0.556 & $3(13)$ & o & 1.000 \\
\hline $\operatorname{HAP}$ group $(n=56)$ & 26 & 30 & & 38 & 18 & \\
\hline ICU mortality & $15(57 \cdot 7)$ & $17(56.7)$ & 0.939 & $23(60.5)$ & $9(50)$ & 0.461 \\
\hline ICU length of stay, day & $22.7 \pm 36.8$ & $14.2 \pm 14.5$ & 0.277 & $21.1 \pm 32.4$ & $12.0 \pm 8.1$ & 0.493 \\
\hline MV duration, day & $19.2 \pm 37.5$ & $10.5 \pm 14.8$ & 0.226 & $18.1 \pm 33.0$ & $6.9 \pm 7.3$ & 0.106 \\
\hline Re-intubation & $3(11.5)$ & $2(6.7)$ & 0.655 & $4(10.5)$ & $1(5.6)$ & 0.662 \\
\hline Tracheostomy & $8(30.8)$ & $9(30)$ & 0.951 & $11(28.9)$ & $6(33 \cdot 3)$ & 0.741 \\
\hline
\end{tabular}

Values are presented as number (\%) or mean \pm SD.

MDR, multidrug-resistant; ICU, intensive care unit; MV, mechanical ventilation; CAP, community-acquired pneumonia; HCAP, healthcare-associated pneumonia; HAP, hospital-acquired pneumonia.

\section{DISCUSSION}

In our study, patients with HCAP and HAP had significantly more severe comorbidity and pneumonia than those of patients with CAP, but the number of pathogens identified did not differ between the pneumonia types. More MDR pathogens were identified in the HAP than those in the CAP and HCAP groups. However, the influence of the pathogen or MDR pathogen identified on the clinical outcomes, such as ICU mortality rate, ICU duration, duration of mechanical ventilation, and frequencies of re-intubation and tracheostomy, was not significant in the entire population or in subgroups ac- cording to the pneumonia classification.

It is controversial whether the pneumonia prognosis differs according to whether the pathogen is identified. In previous studies on the pneumonia prognosis, the severities of underlying disease and comorbidities were evaluated as important prognostic factors. Some HCAP and HAP studies have shown that age, multi-organ dysfunction, septic shock, the Charlson comorbidity index, and SOFA and APACHE II scores are significant prognostic factors for patients with pneumonia $[6,9,17]$. Other studies of patients with severe CAP who required mechanical ventilation or ICU admission reported acute renal failure, septic shock, and the Simpli- 
Table 5. Risk of intensive care unit mortality according to prognostic factors in the entire population

\begin{tabular}{|c|c|c|c|c|}
\hline \multirow{2}{*}{ Prognostic factor } & \multicolumn{2}{|c|}{ Univariate } & \multicolumn{2}{|c|}{ Adjusted } \\
\hline & OR $(95 \% \mathrm{CI})$ & $p$ value & OR $(95 \% \mathrm{CI})$ & $p$ value \\
\hline Age, yr & $1.01(0.99-1.02)$ & 0.595 & $1.04(1.01-1.07)$ & 0.003 \\
\hline Male sex & $0.63(0.32-1.22)$ & 0.168 & $0.59(0.28-1.25)$ & 0.169 \\
\hline APACHE II score & $1.06(1.03-1.09)$ & $<0.001$ & $1.08(1.04-1.12)$ & $<0.001$ \\
\hline SOFA score & $1.20(1.12-1.28)$ & $<0.001$ & $1.31(1.18-1.45)$ & $<0.001$ \\
\hline Charlson comorbidity index & $1.03(0.92-1.14)$ & 0.658 & $1.14(0.99-1.32)$ & 0.066 \\
\hline Pneumonia severity index & $1.01(1.00-1.02)$ & 0.027 & $0.998(0.99-1.01)$ & 0.680 \\
\hline Identified pathogen & $1.21(0.68-2.14)$ & 0.523 & $1.86(0.83-4.17)$ & 0.131 \\
\hline Multidrug-resistant pathogen & $1.39(0.68-2.84)$ & 0.365 & $0.52(0.21-1.29)$ & 0.159 \\
\hline Initial responder to empirical antibiotic therapy & $1.03(0.58-1.83)$ & 0.924 & $0.62(0.33-1.15)$ & 0.131 \\
\hline \multicolumn{5}{|l|}{ Pneumonia classification } \\
\hline Community-acquired pneumonia & 1 (reference) & & 1 (reference) & \\
\hline Healthcare-associated pneumonia & $0.92(0.34-2.48)$ & 0.872 & $0.35(0.10-1.22)$ & 0.100 \\
\hline Hospital-acquired pneumonia & $1.56(0.69-3.55)$ & 0.289 & $1.62(0.53-4.91)$ & 0.395 \\
\hline
\end{tabular}

OR, odds ratio; CI, confidence interval; APACHE, Acute Physiology and Chronic Health Evaluation; SOFA, Sequential Organ Failure Assessment.

fied Acute Physiology Score II as significant prognostic factors $[18,19]$.

In this study, the evaluation of ICU mortality according to the prognostic factors of pneumonia showed that higher APACHE II and SOFA scores were significantly associated with a lower survival rate, but the identification status and the microbe causative of pneumonia were not. This result suggests that the severity of pneumonia or underlying disease may be a more important prognostic factor than microbial etiology, and these data coincide with those of a recent prospective cohort study in Spain, which showed that comorbidities are a stronger determinant of mortality than microbial etiology in a comparison between CAP and HCAP [20].

Several limitations in this study should be mentioned. This study was performed retrospectively in a single tertiary referral hospital; thus, there may have been selection bias because several of the patients enrolled had severe or advanced disease and some patients may have been immunocompromised. The small study population may have limited the statistical power and subgroup analysis. In the pathogen evaluation, MRSA and gram-negative rods may be more easily detected because they are well seen in gram-stained specimens and cultures than anaerobes and viruses. Finally, the initial an- tibiotic therapy and responses were not analyzed in this study.

In conclusion, we found that neither identifying the causative micro-organism nor the existence of a MDR pathogen was associated with the clinical outcomes of pneumonia, including ICU mortality. The severity of the underlying disease or pneumonia itself may be a significant prognostic indicator, regardless of whether the causative micro-organism is identified or if the pneumonia is classified.

\section{KEY MESSAGE}

1. The causative pathogen was identified in $49.0 \%$ of patients with pneumonia treated in the medical intensive care unit (ICU); $21.6 \%$ of patients had a multidrug-resistant pathogen.

2. In the clinical outcomes of pneumonia including ICU mortality, the severity of the underlying disease or pneumonia itself may be a significant prognostic indicator, regardless of the causative micro-organism or the pneumonia classification. 


\section{Conflict of interest}

No potential conflict of interest relevant to this article was reported.

\section{REFERENCES}

1. Richards MJ, Edwards JR, Culver DH, Gaynes RP. Nosocomial infections in medical intensive care units in the United States: National Nosocomial Infections Surveillance System. Crit Care Med 1999;27:887-892.

2. Torres A, Aznar R, Gatell JM, et al. Incidence, risk, and prognosis factors of nosocomial pneumonia in mechanically ventilated patients. Am Rev Respir Dis 1990;142:523528.

3. Kung HC, Hoyert DL, Xu J, Murphy SL. Deaths: final data for 2005. Natl Vital Stat Rep 2008;56:1-120.

4. DeFrances CJ, Lucas CA, Buie VC, Golosinskiy A. 2006 National hospital discharge survey. Natl Health Stat Report 2008:1-20.

5. Labelle AJ, Arnold H, Reichley RM, Micek ST, Kollef MH. A comparison of culture-positive and culture-negative health-care-associated pneumonia. Chest 2010;137:11301137.

6. Tseng CC, Liu SF, Wang CC, et al. Impact of clinical severity index, infective pathogens, and initial empiric antibiotic use on hospital mortality in patients with ventilator-associated pneumonia. Am J Infect Control 2012;40:648-652.

7. Depuydt PO, Vandijck DM, Bekaert MA, et al. Determinants and impact of multidrug antibiotic resistance in pathogens causing ventilator-associated-pneumonia. Crit Care 2008;12:R142.

8. Depuydt P, Benoit D, Vogelaers D, et al. Outcome in bacteremia associated with nosocomial pneumonia and the impact of pathogen prediction by tracheal surveillance cultures. Intensive Care Med 2006;32:1773-1781.

9. Eachempati SR, Hydo LJ, Shou J, Barie PS. Does de-escalation of antibiotic therapy for ventilator-associated pneumonia affect the likelihood of recurrent pneumonia or mortality in critically ill surgical patients? J Trauma 2009;66:1343-1348.
10. Park HK, Song JU, Um SW, et al. Clinical characteristics of health care-associated pneumonia in a Korean teaching hospital. Respir Med 2010;104:1729-1735.

11. Mandell LA, Wunderink RG, Anzueto A, et al. Infectious Diseases Society of America/American Thoracic Society consensus guidelines on the management of community-acquired pneumonia in adults. Clin Infect Dis 2007;44 Suppl 2:S27-S72.

12. American Thoracic Society; Infectious Diseases Society of America. Guidelines for the management of adults with hospital-acquired, ventilator-associated, and healthcare-associated pneumonia. Am J Respir Crit Care Med 2005;171:388-416.

13. Knaus WA, Draper EA, Wagner DP, Zimmerman JE. Apache II: a severity of disease classification system. Crit Care Med 1985;13:818-829.

14. Ferreira FL, Bota DP, Bross A, Melot C, Vincent JL. Serial evaluation of the SOFA score to predict outcome in critically ill patients. JAMA 2001;286:1754-1758.

15. Charlson ME, Pompei P, Ales KL, MacKenzie CR. A new method of classifying prognostic comorbidity in longitudinal studies: development and validation. J Chronic Dis 1987;40:373-383.

16. Fine MJ, Auble TE, Yealy DM, et al. A prediction rule to identify low-risk patients with community-acquired pneumonia. N Engl J Med 1997;336:243-250.

17. Rello J, Lujan M, Gallego M, et al. Why mortality is increased in health-care-associated pneumonia: lessons from pneumococcal bacteremic pneumonia. Chest 2010;137:1138-1144.

18. Tejerina E, Frutos-Vivar F, Restrepo MI, et al. Prognosis factors and outcome of community-acquired pneumonia needing mechanical ventilation. J Crit Care 2005;20:230238.

19. Diaz A, Alvarez M, Callejas C, Rosso R, Schnettler K, Saldias F. Clinical picture and prognostic factors for severe community-acquired pneumonia in adults admitted to the intensive care unit. Arch Bronconeumol 2005;41:20-26.

20. Polverino E, Torres A, Menendez R, et al. Microbial aetiology of healthcare associated pneumonia in Spain: a prospective, multicentre, case-control study. Thorax 2013;68:1007-1014. 